\title{
Graphene As A Hydrogen Storage Material
}

\author{
Nasrin Pourmoghadam ${ }^{1}$, Nezihe Ayas ${ }^{1 *}$ \\ ${ }^{1}$ Eskisehir Technical University, Engineering Faculty, Department of Chemical Engineering, 2 Eylul Campus 26555, Eskisehir, Turkey
}

\begin{abstract}
Although, hydrogen is a promising key solution for the effective delivery of electricity, the issue of finding materials for efficient storage has still remained a significant challenge. Due to the marvelous features of graphene and graphene-based structures, they can be one of the best candidates among all the materials. The purpose of this study was to investigate the synthesis of graphene oxide by improved hummers method and graphene by liquid phase exfoliation (LPE) technique which is safe, easy, economical, and high in quality. In addition, the prepared samples were analyzed and characterized by SEM (Scanning Electron Microscopy), TGA (Thermo Gravimetric Analysis) Setaram Labsys Evo), UV-VIS absorbance spectra, XRD (X-ray diffractometer).
\end{abstract}

\section{Introduction}

Energy demand continues to rise owning to the increasing population of the world and the rapid development of some countries that want to upgrade their living standards. Scientists are looking for alternative energy sources to replace fossil fuels such as solar, wind, hydro, tidal, geothermal, biomass etc. Despite fossil fuels, none of the alternative energy sources can be used directly as liquid or gaseous fuels. Therefore, the above-mentioned energy sources must become the carriers of the energy.

Hydrogen as an energy carrier is the best alternative due to its high energy content of $144 \mathrm{MJ} / \mathrm{kg}$. However, it could not be commercialized due to storage difficulties. Many scientific studies were carried out to develop graphene-based nanomaterial as a green energy resource. Graphene based materials hold the promise for molecular hydrogen $\left(\mathrm{H}_{2}\right)$ storage owning to their ideal binding strength to $\mathrm{H}_{2}$ for room-temperature applications [1].

Hydrogen can be stored in two general methods such as chemical and physisorption. In chemical storage technique which hydrogen is generated through a chemical reaction the material such as ammonia, metal hydrides, formic acid, carbohydrate have been widely used. However, physisorption is a process in which $\mathrm{H}_{2}$ molecules adsorbed at the surface of the porous material such as carbon materials (fullerenes, nanotubes and graphene), zeolites, metal organic frameworks, covalent organic frameworks, micro porous metal coordination materials, clatherates and organotransition metal complexes. Hydrogen on graphene can be attracted by adjusting the distance between adjacent layers. Graphene in comparing with others is cheap and more efficient. Graphene is recognized as a two-dimensional nanomaterial due to its thinness of a single atom, with superior properties formed by the perfect arrangement of covalently bonded carbon atoms in a six-honeycomb lattice. The bond length between carbon-carbon in graphene structure is $0.142 \mathrm{~nm}$. The main superior properties of graphene can be listed as large surface area $\left(2630 \mathrm{~m}^{2} \mathrm{~g}^{-1}\right)$ high electron mobility $\left(200000 \mathrm{~cm}^{2} /(\mathrm{V} \mathrm{s})\right.$ high thermal conductivity $\left(5000 \mathrm{Wm}^{-1} \mathrm{~K}^{-1}\right)$ and high young modulus ( $1100 \mathrm{Gpa})$ [2]. The material finds many application areas such as sensors, transparent electrodes, photodetectors, solar panels, energy storage devices, polymer composites and nano-composites, hydrogen storage, fuel cells, absorbent, catalyst support material, heat transfer material, capacitors, aviation, automotive, membrane in water purification, sensor applications, touch screen, semi-conductors, LCD (Liquid Crystal Displays), energy and batteries and cancer detectors [3]. The usage of graphene is given in Fig.1.

\begin{tabular}{ll}
\hline Usage & Sensors \\
\hline Transparent Electrodes \\
\hline Photodetectors \\
\hline Solar Panels \\
\hline Energy Storage Devices \\
\hline Polymer Composite \\
\hline Fuel Cell \\
\hline Absorbent \\
\hline Catalyst Supported Material \\
\hline Heat Transfer Material \\
\hline Capacitors \\
\hline Automotive \\
\hline Membrane In Water Purification \\
\hline Touch Screen \\
\hline Semi-conductors \\
\hline Lcd \\
\hline Cancer Detectors \\
\hline
\end{tabular}

Fig.1. Application of graphene.

\footnotetext{
Corresponding author: nazcan@eskisehir.edu.tr
} 
In the term of synthesis methods, lots of research has been done with the aim of finding optimization technique. Some of the main graphene fabrication methods include (a) Chemical Exfoliation methods in which use strong acid with the aim of exfoliation of graphene from graphite, (b) Chemical vapor deposition (CVD) in which product is formed on a heated substrate by a chemical reaction, (c) Epitaxial growth on $\mathrm{SiC}$ method is carried out at high temperature, and (d) Liquid phase peeling that is safe, high quality and easy [4]. In addition, this route allows producing graphene nanomaterials at a large-scale for applications such as nanocomposite, transparent electrode, and sensors. Since the quality of graphene nanomaterials are strongly relies on morphology, composition, size and also the surface area, different types of characterization methods have been applied which are: X-ray (XRD, XPS, EDX) spectroscopy, electron microscopy (TEM, SEM), visible spectroscopy, Scanning Probe Microscopy (AFM, STM), and Raman Spectroscopy which helps to determine ordered and disordered crystal structures [5].

Several hydrogen storage methods have been investigated so far. The efficiency of storage is measured by two factors, Gravimetric density (GD), which is defined as the amount of energy stored in a given space region per unit volume, and the second is the volumetric density (VD) defined as the mass of hydrogen per unit volume.

In addition, the importance of graphene as the future of hydrogen storage technology is increasing day by day due to its marvelous properties such as electrical conduction, durability and flexibility. Graphene nano structure is produced from the exfoliation method in a large scale and cost-effective way. For instance, graphene nanoflakes can be produced more than several micrometers in sizes at high concentration by liquid phase exfoliation [6].

Chemical exfoliation methods are favorable for graphene-based materials. This cost effective method classified to liquid phase exfoliation method and graphite oxide route such as improved hummers methods. Briefly, in liquid exfoliation methods, The natural graphite flake powder was dispersed in an appropriate solvent. Next, sonication was applied to sample at room temperature. Followed by centrifugation then dried in oven. Graphene samples prepared by liquid phase exfoliation are free from residual oxygen and they contain much lower structural defects in comparison with hummers method. The major drawbacks of this method are low graphene concentrations and lateral sizes are about several hundred nm of graphene powders which is because of sonication. Extended sonication times $(>400 \mathrm{~h})$ were shown to boost graphene concentration which makes it impractical for large scale.

By graphite oxide route method producing of graphene oxide (GO) and reduced-graphene oxide (r-GO) from graphite powders, is possible by using oxidizing agents such as strong acids. In order to exfoliate graphite oxide to graphene, sonication was applied in appropriate solvent.
In this study, two different methods such as improved hummers and liquid phase exfoliation were used to obtain graphene and graphene oxide using graphite.

\section{Methods}

\subsection{Graphene Synthesis}

\subsubsection{Synthesis by liquid phase exfoliation method}

The natural graphite flake, (325 mesh) (Alfa Aesar) was dispersed in Isopropyl alcohol (IPA, Merck Emsure) at the ratio of $3 \mathrm{mg} / \mathrm{mL}$. All the material was used without any further purification and the solvent was selected due to the low boiling point $\left(82.5^{\circ} \mathrm{C}\right)$ property which allows to obtain graphene-based nano-materials with high concentration. Next, the sample was sonicated $(35 \mathrm{kHz}$, $100 \mathrm{~W}$ ) for $90 \mathrm{~min}$. followed by centrifuging at $500 \mathrm{rpm}$ for $45 \mathrm{~min}$ then dried at $105^{\circ} \mathrm{C}$ [7]. The properties of the obtained graphene were determined by some characterization techniques (Fig.2).

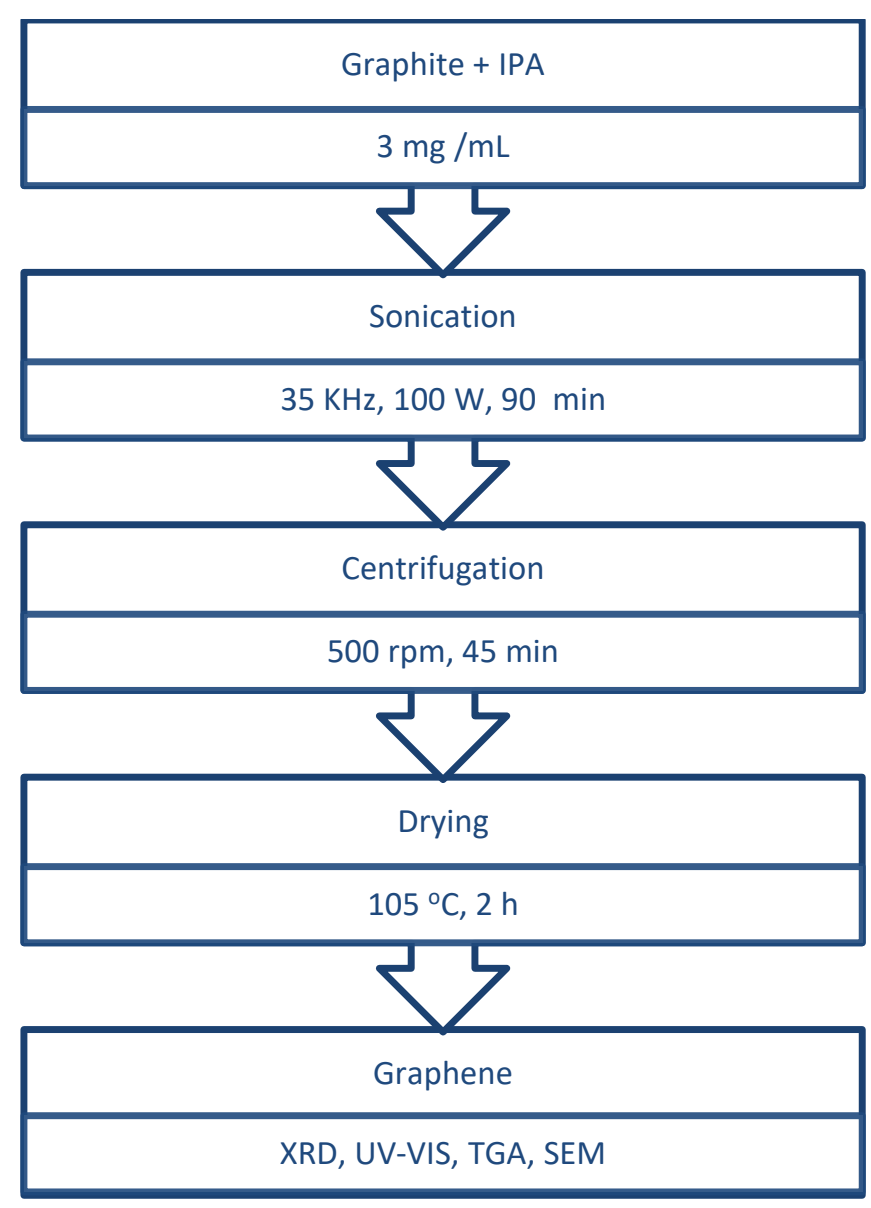

Fig.2. Schematic representation of liquid phase exfoliation (LPE) method by IPA.

\subsubsection{Graphene oxide Synthesis by improved hummers method}


To produce graphene oxide by improved hummers method [7], a specific amount of graphite powder (3 g) was mixed with an acidic solution of $\mathrm{H}_{2} \mathrm{SO}_{4}: \mathrm{H}_{3} \mathrm{PO}_{4}$ (in the ratio of 360:40 mL) under stirring in an ice bath . Next, 18 $\mathrm{g}$ of $\mathrm{KMnO}_{4}$ was carefully added to the dispersion step by step. The mixture was stirred overnight at $50{ }^{\circ} \mathrm{C}$. When the mixture was cooled to room temperature, $400 \mathrm{~mL}$ of distilled water (DW) and then about $5 \mathrm{~mL}$ of $\mathrm{H}_{2} \mathrm{O}_{2}$ were added into the mixture in order to stop the reaction. The obtained solution was centrifuged and washed several times until reaching a neutral $\mathrm{pH}$. Finally, it was vacuumdried at $50{ }^{\circ} \mathrm{C}$ overnight to give graphite oxide powder. In order to exfoliate flakes into graphene oxide sheets, the graphite oxide powder was dispersed in water $(1 \mathrm{mg}$. $\left.\mathrm{mL}^{-1}\right)$ and subjected to ultrasonication $(1200 \mathrm{~W})$ for 90 $\min$.

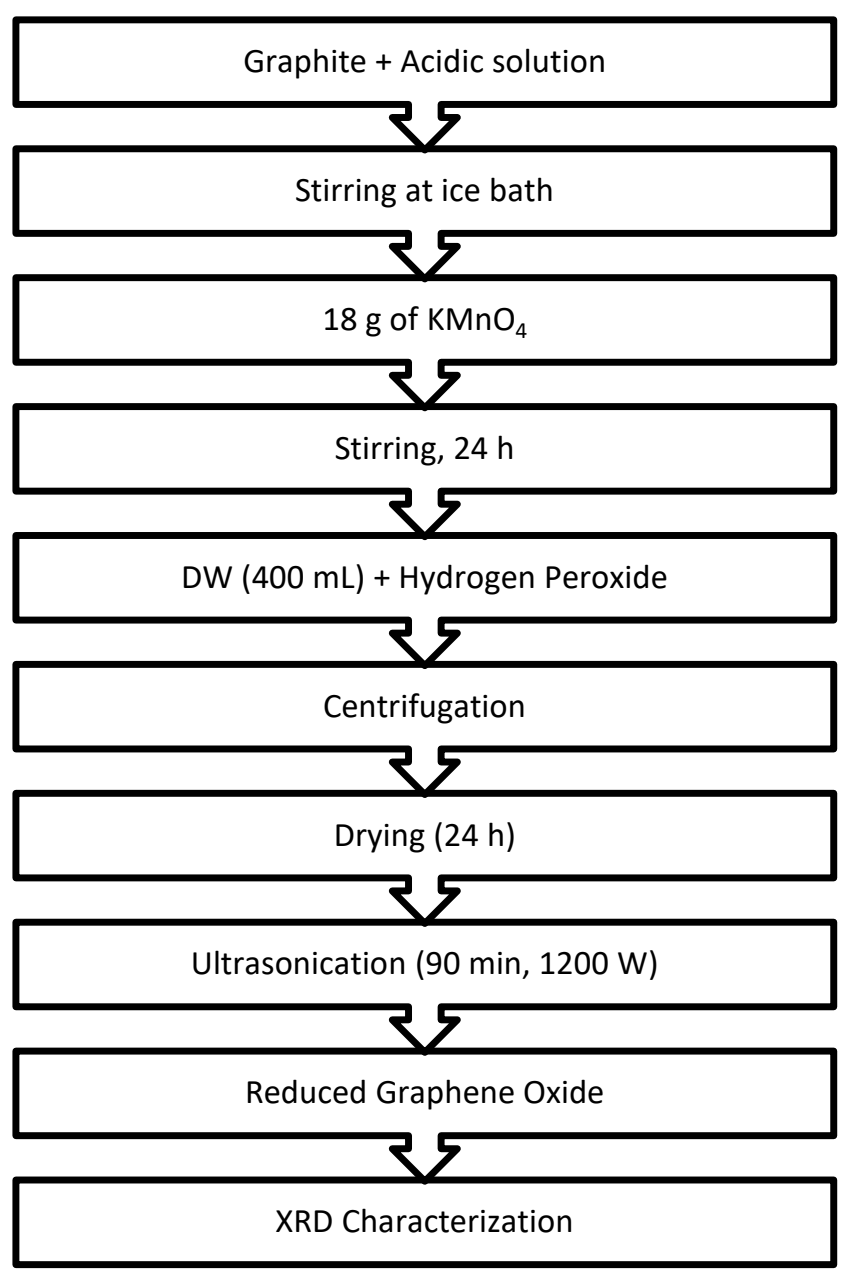

Fig.3. Schematic representation of improved hummers method.

\subsection{Characterization}

$\mathrm{X}$-ray diffraction technique was recorded by using Rigaku XRD device and data was collected from $2 \theta=0$ to $70^{\circ}$ with a scanning rate of $2 \mathrm{deg}$. $\mathrm{min}^{-1}$. The X-ray diffraction of graphene and graphite are given in Fig.4. There are two significanat peaks around $27^{\circ}$ and $54^{\circ}$ were appointed to the (002) plane (the usage of this parameter is calculation of lattice parameter of gamma and gamma prime). The graphene indicates almost the same diffraction peaks as graphite with different intensity. The result obtained because of the size of the layer became reduced during exfoliation of graphene. The outcome is in good agreement with literature [8].

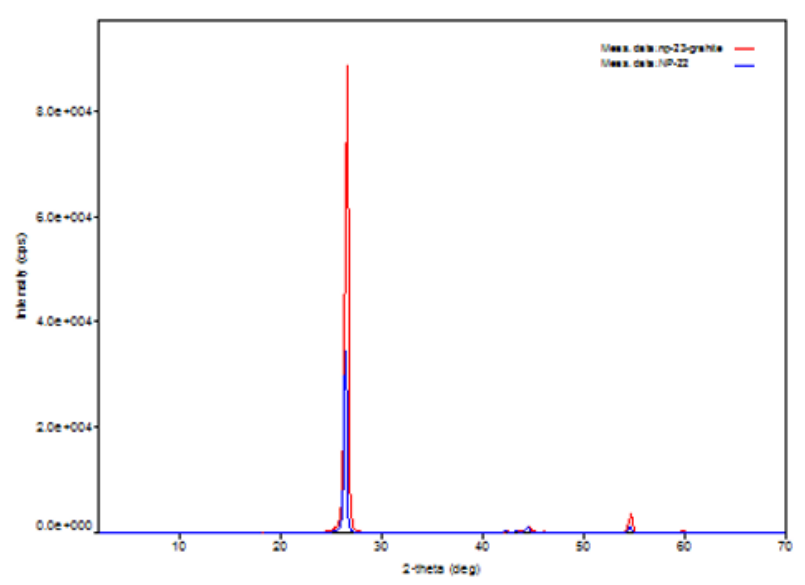

Fig.4. X-ray diffractometer pattern of graphite and graphene obtained by liquid phase exfoliation.

In addition, the result of obtained graphene oxide by the improved hummers method is shown in Fig. 5. In this pattern, a sharp peak is found around $25^{\circ}$ and $42^{\circ}$. L. Stobinski et al. performed the synthesis of reduced graphene oxide and it was determined that the graphene oxide obtained was compatible with the XRD pattern in our study [9].

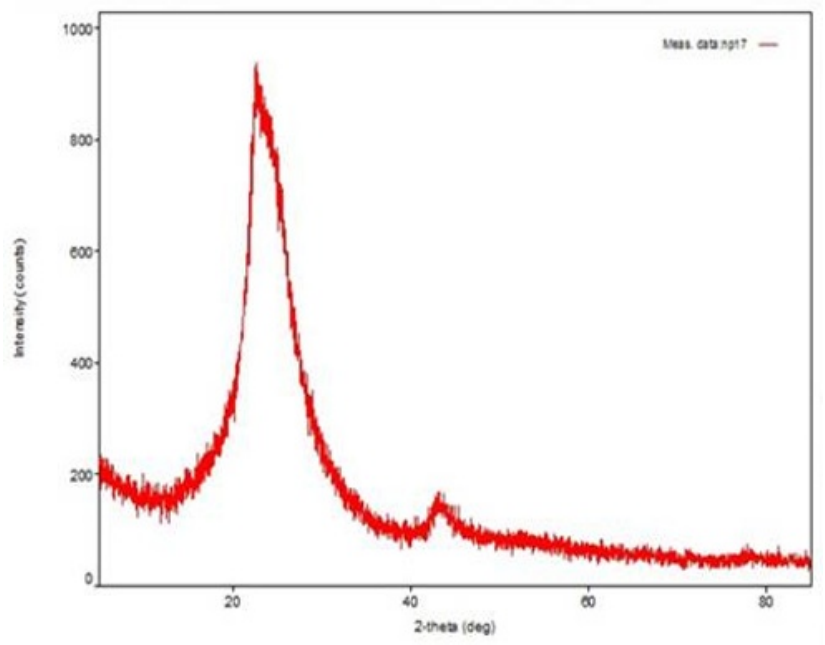

Fig.5. X-ray diffractometer pattern of reduced graphene oxide obtained by using improved hummers method.

UV-VIS absorbance spectra of the graphene-based dispersions and the pure solvents were performed in the 200-800 nm wavelength range using Varian Cary100 Bio spectrometer with $1 \mathrm{~cm}$ cuvettes. According to Fig. 6 The only peak that was found in UV-VIS absorption spectrum of dispersion was approximately at $264 \mathrm{~nm}$ which is characteristic to graphene and can be credited to aromatic $\mathrm{C}=\mathrm{C}$ bonds. Moreover, Y.Celik et al. found the UV-VIS absorption spectrum of graphene to be flat except for the peak at $264 \mathrm{~nm}$ [10]. 


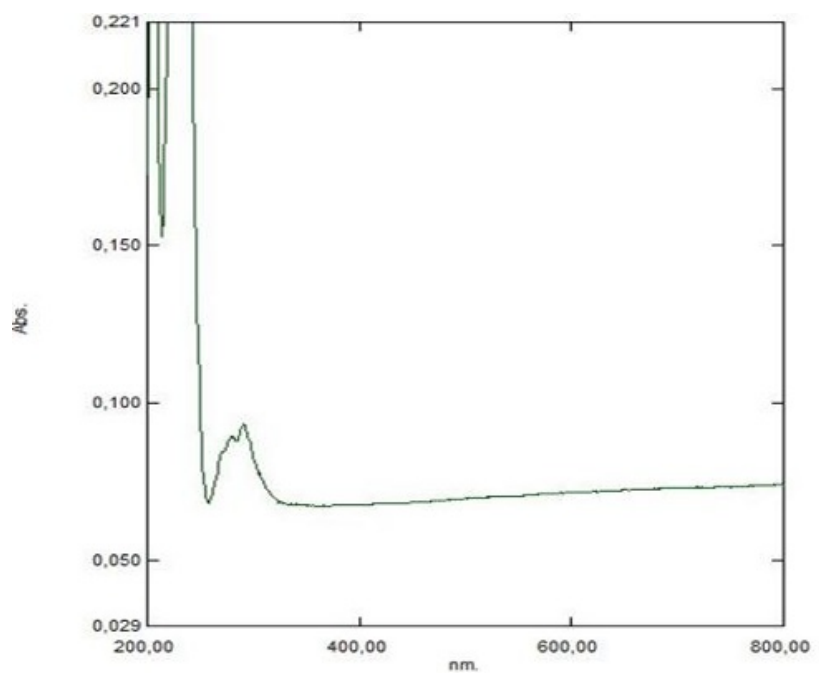

Fig.6. UV-Vis absorption spectra of graphene obtained from liquid phase exfoliation.

SEM is one of the most popular of the analytical methods in order to investigate shape and morphology of materials. Fig.7 displays agglomeration of the exfoliated sample in low boiling point sample for $90 \mathrm{~min}$ followed by centrifugation. The agglomerated structure could not be prevented entirely due to Van der Waals forces between layers. SEM analysis of the solid product was performed by dropping some sample onto a substrate and then drying in an oven at $80^{\circ} \mathrm{C}$ in air.

The result seems to be compatible with the sem images in the literature [10-11].

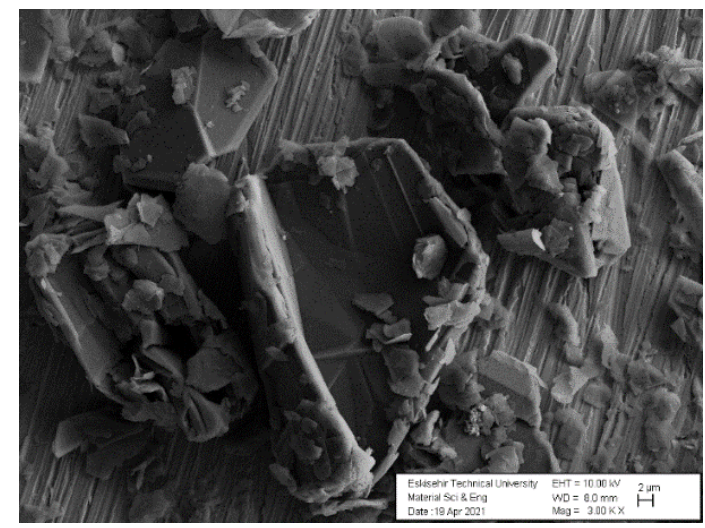

Fig.7. Sem pictures of graphene obtained from liquid phase exfoliation.

The 3000x magnification of the SEM image indicates a smoother buildup and is visibly reduced by the process of exfoliation and the structure of graphene nano sheets consists of multiple layers (Fig.7).

TGA experiments were performed with the samples having a mass of $10 \pm 1 \mathrm{mg}$ in a $100 \mu \mathrm{L}$ alumina crucible. The sample was heated from $30^{\circ} \mathrm{C}$ to $850{ }^{\circ} \mathrm{C}$ at a heating rate of $10{ }^{\circ} \mathrm{C} \mathrm{min}{ }^{-1}$ and under a nitrogen flow of 20 $\mathrm{mL} \cdot \mathrm{min}^{-1}$. The weight loss curve, indicates just one decreased step which starts at approximately $200^{\circ} \mathrm{C}$ and can be due to loss of oxygenated groups that may be present in the graphene, R. Hack et al. reported the same result [12].

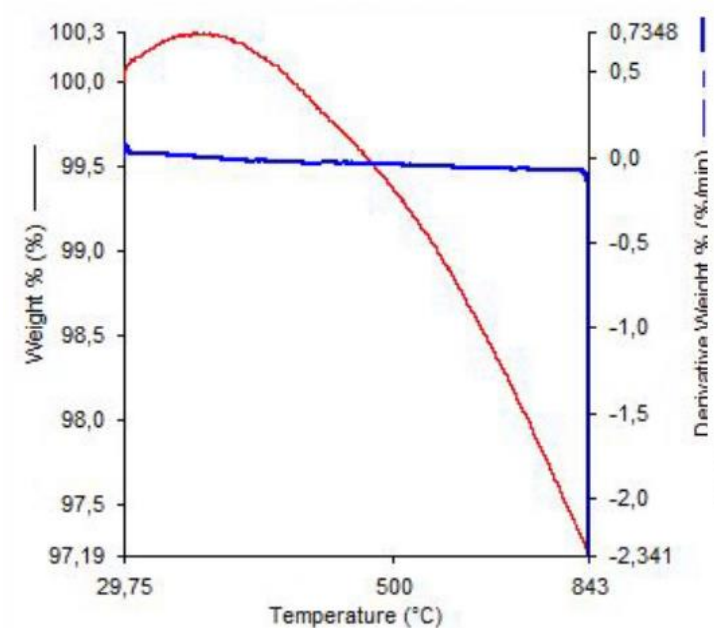

Fig.8. Thermogravimetric analysis (TGA) curve of graphene obtained from liquid phase exfoliation.

\section{Result and discussion}

As a result, two various techniques were investigated to synthesis graphene-based nanomaterials. Hummers method usually gives high-yield graphene however it suffers from defects and impurities in graphene structure. Liquid phase exfoliation (LPE) method is safe, easy, economical, and high in quality.

\section{Conclusion}

Despite many achievements and studies, yet there is a distinct need for further investigation. Hence, researcher should focus on fabricate and characterize cost-effective graphene-based materials substituting expensive noble metals with cheaper and easily abundant one which can be employed at industrial scale because almost all preparation of nanostructured graphene-based materials has been done at the laboratory scale.

Future work for liquid phase exfoliation method, optimization studies can be investigated to increase graphene concentration and to obtain uniform layer of graphene, and to decrease the agglomeration. The exfoliation studies can be improved using alternative dispersion methods such as milling, shear mixing, different solvents, and different quality of graphite as a starting material.

\section{References}

1. V. Jain, B. Kandasubramanian, J. Mater. Sci. 55, 1865-1903 (2020)

2. C. Soldano, A. Mahmood, E. Dujardin, J. Carbon, 48, 2127-2150 (2010)

3. P. Jospha, G. Patrick, M. Thaddeus, Mat. Sci. Eng. 215, 9-28, (2017)

4. G. Wang, J. Yang, J. Park, X. Gou, B. Wang, H. Liu, J. Yao, J. Phys. Chem. C, 112, 22, 8192- 8195 (2008)

5. E. Rommozzi, M. Zannotti, R. Giovannetti, C. Amato et al, Catalyst J. 08-00598 (2018) 
6. V. Tozzini, V. Pellegrini, Phys. Chem. 15, 80 -89 (2013)

7. R. Zafarghandi, A. Hadi, J. Karimi Sabet, Flat. Chem. 74-48 (2017)

8. X. Wang, L. Zhang, RSC. Advance, Issue 7 (2019)

9. L. Stobinski et al, Electron Spec. and Rel. Phe. 249, 145-154 (2014)
10. Y. Çelik, E. Flahaut, E. Suvacı, Flat. Chem. 1, 74-88 (2017)

11. R. Siburian, H. sihotang, S. Lumban Raja, M. Supeno, C. Simanjuntak, Chem. 34, 182-187 (2017)

12. R. Hack et al, Rio de Janeiro, 23, 1517-7076 (2018) 